\title{
$X X$. On the use of the biquartz in determining the position of the plane of polarization
}

\section{A. W. Ward}

To cite this article: A. W. Ward (1889) XX. On the use of the biquartz in determining the position of the plane of polarization, Philosophical Magazine Series 5, 28:171, 134-138, DOI: 10.1080/14786448908619843

To link to this article: http://dx.doi.org/10.1080/14786448908619843

曲 Published online: 08 May 2009.

Submit your article to this journal

Џ Article views: 2

Q View related articles $₫$ 


$\left.\begin{array}{cc|cc}\text { Volts in primary, } & 128 & \text { Current in primary, } & 14 \cdot 2 \\ \text { " } & \text { secondary A, 119 } & " & \text { secondary A, 8.0 } \\ \text { " } & \text { secondary B, 119.5 } & " & \text { secondary B, 8.2 }\end{array}\right\} 16 \cdot 2$

Connecting up the secondaries of transformers $A$ and $B$ in parallel, the volts at the terminals of the primary being the same as before, and the secondary circuit being lamps.

\begin{tabular}{cc|cc} 
Volts in primary, & 128 & Current in primary, & $17 \cdot 4$ \\
$\#$ secondary, & 121 & $y$ secondary, & $16 \cdot 3$
\end{tabular}

Here we have to give $17 \cdot 4$ amperes to the primary instead of $14 \cdot 2$ to get same current in secondary, and the volts in secondary are practically the same as before.

If we were being supplied with electricity, what should the meter measure? Surely the amount of energy we use. But ordinary meters only measure ampere-hours, and so caunot but give records in favour of consumer or supplier. The sooner the public understand this, the sooner we shall have a scientific meter in our houses.

$\mathrm{XX}$. On the Use of the Biquartz in determining the position of the Plane of Polarization. By A. W. WARD*.

YTHE biquartz has been so often used, especially on the 1 Continent, by investigators on the rotation of the plane of polarization of light, and with such extremely varying degrees of accuracy, that it seems of interest to account for these results mathematically. Verdet and $H$. Becquerel obtained results which varied by less than $4^{\prime}$; while Wertheim, Matteucci, Edlund, Lüdtge, and Villari obtained results varying by as much as $2^{\circ}$. Lüdtge has in one case obtained a rotation of $4^{\circ}$ where, on his own showing, the light was circularly polarized. Verdet's and Becquerel's accurate results were obtained when rotation was loolsed for in liquids and isotropic substances; and the inaccurate results of Wertheim \&c. were obtained when seeking for a rotation in doublyrefracting substances. In the former case the light remained plane-polarized, in the latter it became elliptically-polarized, and the position of the plane of polarization was really that of one of the axes of the ellipse. In the present investigation we shall then determine with what degree of accuracy the biquartz can be used to determine the position of the axes in elliptically-polarized light.

Let us suppose that the elements of the elliptically-polarized

* Communicated by the I'hysical Society: read June 8, 1889. 
light are given by the displacements along the axes of the ellipse, and by the inclination of an axis of the ellipse to some direction fixed in space. Let the displacements $\xi$ and $\eta$ be parallel to the axes of the ellipse, and let the axes of $x$ and $y$ be fixed in space, $z$ being the axis along which the light travels; and let $\omega$ be the angle between the axes of $x$ and $\xi$. If then $c^{2}$ be the intensity of the light, $\tan \gamma$ the ratio of the axes of the ellipse, the vibrations of the light are given by the equations

$$
\left.\begin{array}{l}
\xi=c \cos \gamma \cos \frac{2 \pi}{\lambda}(v t-z), \\
\eta=c \sin \gamma \sin \frac{2 \pi}{\lambda}(v t-z) .
\end{array}\right\} . . \cdot
$$

The angles $\gamma$ and $\omega$ are known whenever we know the history of the light; how it became converted from planepolarized into elliptically-polarized. If, for instance, tho change took place in passing througb a doubly refractive medium whose axes are those of $x$ and $y$, then

$$
\left.\begin{array}{r}
\tan 2 \omega=\tan 2 \alpha \cos \beta, \\
\sin 2 \gamma=\sin 2 \alpha \sin \beta,
\end{array}\right\} \quad \text {. . . . }
$$

where $\beta$ is the total angular retardation, and $\alpha$ the inclination of the initial plane of vibrations to that of $x z$. In these equations $\beta$ is a function of $\lambda$, viz. $\frac{2 \pi}{\lambda}\left(\mu_{1}-\mu_{2}\right) z$, where $\mu_{1}$ and $\mu_{2}$ are the indices of refraction along the axes of $x$ and $y$ respectively. If $\alpha$ is small, variations in $\omega$ due to $\lambda$ are not important; but if $\alpha$ is large this is no longer the case, as we shall eventually see.

Let us now pass the light (1) through a biquartz which is such that the plane of polarization of light, of wave-length $\lambda$, is turned through an angle $\phi$. This rotation will simply turn the ellipse as a whole, and not affect the ratio of the axes. Hence for upper half of the biquartz $\omega$ becomes $\omega+\phi$, and for lower half $\omega-\phi$.

Let the light be now analysed by a Nicol whose plane of vibrations makes an angle $\theta$ with the plane of $x z$. If then $l^{2}$ be intensity of light passing through the upper half of biquartz, and $k^{2}$ that of light passing through the lowor half,

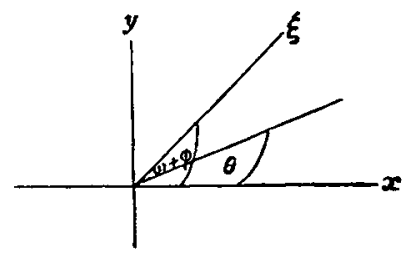
we have, as ysual, 


$$
\left.\begin{array}{l}
h^{2}=c^{2} \cos ^{2} \gamma \cos ^{2} \overline{\omega+\phi-\theta}+c^{2} \sin ^{2} \gamma \sin ^{2} \overline{\omega+\phi-\theta}, \\
k^{2}=c^{2} \cos ^{2} \gamma \cos ^{2} \overline{\omega-\phi-\theta}+c^{2} \sin ^{2} \gamma \sin ^{2} \overline{\omega-\phi-\theta} ;
\end{array}\right\}
$$

or

$$
\left.\begin{array}{l}
h^{2}=\frac{c^{2}}{2}\{1+\cos 2 \gamma \cos 2(\omega+\phi-\theta)\}, \\
k^{2}=\frac{c^{2}}{2}\{1+\cos 2 \gamma \cos 2(\omega-\phi-\theta)\} .
\end{array}\right\} . . .
$$

We have now to determine what value of $\theta$ makes $h^{2}=k^{2}$ for all values of $\lambda$.

Equating $h^{2}$ to $k^{2}$, we get

$$
\cos 2 \gamma \sin 2 \phi \sin 2 \overline{\omega-\theta}=0 . \quad \text {. . . . }
$$

This equation is satisfied whenever

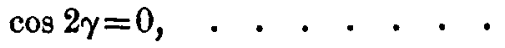

$$
\begin{aligned}
& \sin 2 \phi=0, \quad . \quad . \quad . \quad . \quad . \\
& \sin 2(\omega-\theta)=0 \text {. . . . . . }
\end{aligned}
$$

The first of these solutions occurs when $\gamma=\frac{\pi}{4}, i$. e. when the elliptically-polarized light is really circularly polarized. In this case the phrase plane of polarization has no meaning at all, and so it need not be discussed.

The second solution (7) gives $\phi=\frac{\pi}{2}$. This can only be the case for one particular wave-length, and depends simply on the thickness of the biquartz. A biquartz is usually made of such a thickness that $\phi$ is $\frac{\pi}{2}$ for the yellow light from the brightest part of the spectrum. We shall suppose this to be the case here.

The third solution gives

$$
\omega=\theta .
$$

If, then, this solution does not hold for all valnes of $\lambda$, then, however the analysing Nicol be turned, both halves of the biquartz can never be made of the same uniform tint.

Now, considering the equation

$$
\tan 2 \omega=\tan 2 \alpha \cos \beta,
$$

we see that $\omega=\alpha$ always if $\beta=0$, that is, if the incident planepolarized light always remains so. If $\beta$ is not equal to 0 , then still $\omega=\alpha$ for all values of $\lambda$, if $\alpha=0$ or $\frac{\pi}{4}$. If $\alpha$ is 0 , then 
$\gamma=0$, and the light is plane-polarized as before. The case we have to discuss, then, is $\alpha=\frac{\pi}{4}$.

If $\alpha=\frac{\pi}{4}$, and also for any particular value of $\lambda, \beta=\frac{\pi}{4}$, then light of that colour is circularly-polarized. Hence, howerer wo alter $\theta$, no change in the intensity of that light will take place. If this circularly-polarized light comes from a prominent part of the spectrum, it will be impossible to note small change in the tint of passage due to the varying presence of other colours. The difficulty experienced will be precisely similar to that of fixing the position of the plane of polarization by means of the yellow tint of passage instead of the violet tint.

If $\beta$ is never so great as $\frac{\pi}{2}$, then, when both halves of the biquartz are of the same uniform tint, the position of the analyser determines the position of the ellipse; but the uniform tint will not be that due to excluding the yellow light of the spectrum, but will contain lights of every colour, but not in that proportion which constitutes white light. The tint may be rosy or yellow.

If $\alpha$ is neither 0 nor $\frac{\pi}{4}$, then $\omega$ varies with $\lambda$, and cannot possibly satisfy the solution for all values of $\theta$. In this case, then, both halves of the biquartz can never be made of the same tint. As this is the general case, we conclude that the biquartz is not a suitable instrument to use when, instead of plane-polarized light, we have elliptically-polarized.

The following table gives the values of $\omega$ due to variations in $\alpha$ and $\lambda$ when the light has passed through a quarter undulation-plate of quartz. The values have been calculated from Rudberg's table of indices, quoted on p. 317 of Glazebrook's 'Optics.' The capital letters refer to the lines of the spectrum.

\begin{tabular}{|c|c|c|c|c|c|}
\hline & & O. & D. & E. & G. \\
\hline & $\beta$. & $80^{\circ}$. & $90^{\circ}$. & $100^{\circ}$. & $126^{\circ}$. \\
\hline$\omega=10^{\circ}$. & $\omega$ & $2^{\circ}$ & 0 & $-2^{\circ}$ & $-6^{\circ}$ \\
\hline$*=20^{\circ}$. & $\omega$ & $4^{\circ}$ & 0 & $-4^{\circ}$ & $-13^{\circ}$ \\
\hline$\omega=44^{\circ}$. & $\omega$ & $39^{\circ}$ & 0 & $-39^{\circ}$ & $-43^{\circ}$ \\
\hline
\end{tabular}

The above is simply given as an illustration of the magnitude of the quantities involved in a particular case where it is easy to make the calculations. I have tried the experiment 
by passing light the reverse way through an elliptic analysor (i. e. a Nicol prism and quarter undulation-plate), then through the biquartz, and finally through an analyser. It is found quite impossible to get any match between the two halves of the biquartz when $\alpha$ is large. The actual dispersion of the axes depends upon the variations of $\beta$ with $\lambda$, and this is very much greater in quartz than in such a doubly refracting substance as compressed glass. But in most cases there will always be sufficient variation to make the use of the hiquartz a very unsuitable method, and this does, I think, account for the two classes of results mentioned at the beginning of this paper.

In conclusion, I have only to express my gratitude to $\mathrm{Mr}$. Glazebrook for many valuable suggestions, and to Professor Thomson for the use of the Cavendish Laboratory.

XXI. Notices respecting New Books.

$A$ Treatise on Spherical Trigonometry, and its application to Geodesy and Astronomy; with numerous examples. By Dr. J. CASEY, F.R.S. (Longmans : 1889.)

DR. CASEY has "struck oil" as a writer of Mathematical

Text-books. It is not so many years since he began this career with his useful and excellent 'Sequel to Euclid,' which has now reached a fifth edition, and since that time he has produced other text-books of like good quality. Having given us an "elementary" and a more advanced Plane Trigonometry, he now completes this special corner of mathematical literature with the work before us. The student will find here all, or nearly all, he wants in a text-book on the subject, illustrated by much matter selected from foreign periodicals, with variety of proofs. Following a practice which has come much into vogue of late years, many results are ticketed with the names of the earliest publishers of thern: for instance, two formulw which frequently occur in the solution of triangles are called the first and second Staudtans of a triangle. Recent points and lines which occur in Plane Geometry, and which have analogues in Spherics, have the like names bere: some, as the Lemoine point and the Symmedian point, which are identical in plane, do not coincide in Solids. The specially noteworthy chapters, as might be expected from Dr. Casey's original work in this field, are, in our opinion, those upon the small Circles on the Sphere and on Inversions. There is a large collection of exercises, and, after the author's previous manner, the more noteworthy results are numbered; of these, 495 are given. In addition to a handy and compact account of the purely Trigonometrical details, there is a final chapter on the applications to Geodesy and Astronomy. The text is accompanied by a short index. 